\title{
Limited translation invariance of human visual pattern recognition
}

\author{
MARCUS DILL and MANFRED FAHLE \\ Universitäts-Augenklinik Tübingen, Tübingen, Germany
}

\begin{abstract}
Visual object recognition is considered to be largely translation invariant. An earlier study (Foster \& Kahn, 1985), however, has indicated that recognition of complex novel stimuli is partially specific to location in the visual field: It is significantly easier to determine the identity of two briefly displayed random patterns if both stimuli are presented at the same, rather than at different, locations. In a series of same/different discrimination tasks, we characterize the processes underlying this "displacement effect": Horizontal and vertical translations are equally effective in reducing performance. Making the task more difficult by increasing pattern similarity leads to even higher positional specificity. The displacement effect disappears after rotation or contrast reversal of the patterns, indicating that positional specificity depends on relatively low levels of processing. Control experiments rule out explanations that are independent of visual pattern memory, such as spatial attention, eye movements, or retinal afterimages. Positional specificity of recognition is found only for same trials. Our results demonstrate that position invariance, a widely acknowledged property of the human visual system, is limited to specific experimental conditions. Normalization models involving mental shifts of an early visual representation or of a window of attention cannot easily account for these findings.
\end{abstract}

Our brain is capable of recognizing a learned object even if this object is presented under conditions producing a considerably different retinal input. To an impressive extent, visual memory is invariant for changes in size (Biederman \& Cooper, 1992; Bundesen \& Larsen, 1975; Fiser \& Biederman, 1995; Kolers, Duchnicky, \& Sundstroem, 1985), orientation in two and three dimensions (Arnoult, 1954; Bülthoff \& Edelman, 1993; Logothetis, Pauls, Bülthoff, \& Poggio, 1994; Rock, 1973), and position in the visual field (Biederman \& Cooper, 1991; Ellis, Allport, Humphreys, \& Collis, 1989). Closer examination in the last three decades has revealed the limits of perceptual invariances, for example, in the recognition of novel stimuli (Bülthoff \& Edelman, 1992, 1993; Edelman \& Bülthoff, 1992; Foster, 1978, 1984; Logothetis et al., 1994; Nazir \& O'Regan, 1990). Even familiar objects or faces are hardly recognizable under extreme transformations and when presented from an unfamiliar viewpoint (Kolers et al., 1985; Thompson, 1980; Troje \& Bülthoff, 1996). A significant bias exists in the literature: While, for example, invariance for 2-D and 3-D rotations has attracted much scientific attention during the last decades, there have been only a few psychophysical studies done on how our visual system can determine the identity of a stimulus

M.D. was supported by Boehringer Ingelheim Fonds; M.F. was supported by the von Humboldt Society (Max Planck Prize) and by Deutsche Forschungsgemeinschaft (SFB 307). We thank E. Bricolo, S. Edelman, B. Olshausen, and T. Poggio for valuable discussions, and Constantine Papageorgiou for correcting the English. Comments and suggestions by L. E. Krueger, M. Peterson, T. Sanocki, and an anonymous reviewer helped to improve the manuscript considerably. Correspondence can be addressed to M. Dill, Universitäts-Augenklinik, Sektion Visuelle Sensorik, Waldhörnlestr. 22, D-72072 Tübingen, Germany (e-mail: marcus.dill@uni-tuebingen.de). independently of its location in the retinal image, a phenomenon known as position or translation invariance.

Introspectively experienced position invariance is contaminated by our highly developed ability to fixate objects of interest. Insensitivity to displacements in the visual field may be mimicked by saccade-mediated tolerance for translations in absolute space. However, position invariance is more than self-delusion, given a considerable amount of evidence from electrophysiological studies: Starting with complex cells in V1 (Hubel \& Wiesel, 1962), neurons in many parts of the visual system respond to a specific stimulus irrespective of its location in the receptive field. On the basis of this observation, it has been proposed that position invariance might be the result of a step-by-step projection of locally translation-tolerant elements to cells with receptive field sizes gradually increasing in higher visual areas (Földiák, 1991; Fukushima, 1980; Rolls, 1992). Indeed, neurons in inferotemporal (IT) cortex, a brain area highly involved in shape recognition and shortterm pattern memory, have receptive fields up to $50^{\circ}$ in diameter and receive information from a large part of the visual field. The selectivity of many IT cells for highly specific features, even faces or hands, is largely independent of the stimulated location within their receptive fields (Ito, Tamura, Fujita, \& Tanaka, 1995; Logothetis, Pauls, \& Poggio, 1995; Schwartz, Desimone, Albright, \& Gross, 1983; Tovee, Rolls, \& Azzopardi, 1994). However, many cells in IT-especially in its posterior parthave smaller receptive fields covering only a few degrees of the visual field, and some show variations in stimulus selectivity (Ito et al., 1995; Logothetis et al., 1995). Even for IT cells with stimulus selectivity largely preserved throughout the receptive field, absolute firing levels may vary with retinal location (Schwartz et al., 1983). The sit- 
uation is further complicated by the possibility that a given pattern stimulates more than one cell and is represented by the combined activity of a number of neurons (Fujita, Tanaka, Ito, \& Cheng, 1992; Tanaka, Saito, Fukada, \& Moriya, 1991; Wachsmuth, Oram, \& Perrett, 1994). Despite rather large receptive fields, an individual cell's contribution to the whole system's performance might be reduced or increased by a displacement in the retinal image. All these findings and considerations make it difficult to predict psychophysical performance from translation tolerance of individual IT cells.

Behavioral data from animal and human research are scarce. Only for insects do they tell a clear story in that, in these small-brain animals, pattern memory is specific for retinal location (Collett, 1992; Dill \& Heisenberg, 1995; Dill, Wolf, \& Heisenberg, 1993; Heisenberg, 1995; Wehner, 1981). In vertebrates, on the other hand, position-invariant recognition has been reported episodically in a few species and with only small sets of very simple stimuli (Cerella, 1990; Cronly-Dillon, Sutherland, \& Wolfe, 1966; Myers, 1955). In human psychophysical studies, translation invariance turned out to be either complete or partial, depending on stimuli and tasks involved. Biederman and Cooper (1991) and Ellis et al. (1989) showed that retinal displacements had no effect on naming latency in a visual priming paradigm. Their subjects had to respond to line drawings or photographs of familiar objects, such as birds or pianos-objects that observers had probably experienced hundreds or even thousands of times under a variety of conditions. Hence, it is not possible to determine whether translation invariance is achieved through decades of stimulus-specific learning or by some mechanism intrinsic to the recognition process.

Unraveling the processes underlying translation invariance, therefore, requires the employment of novel, unfamiliar visual patterns. Other than for 3-D orientation (Bülthoff \& Edelman, 1993), this has only rarely been done in investigations on the role of visual-field position: Nazir and O'Regan (1990) found that subjects trained to discriminate a target dot pattern from two distracting stimuli at one location of the visual field often did not recognize the learned pattern at new retinal positions. A number of observers, however, achieved partial or even full discrimination performance after displacement for some of the stimuli. Since they reported "recognition" of abstract features (such as, e.g., a "bizarre telephone") in individual dot patterns, the authors concluded that tolerance for translations depended on high-level associations (O'Regan, 1992). If no abstract descriptor exists for a certain stimulus, it must be stored by a positionspecific type of memory or else is lost. Whether partial invariance can be explained by feature extraction alone and how the possible retinotopic backup memory can be characterized has yet to be examined.

Positional specificity has also frequently been reported for a variety of other perceptual learning tasks, ranging from vernier acuity learning to the improvement of texture discrimination (Fahle, 1994; Fahle, Edelman, \&
Poggio, 1995; Fiorentini \& Berardi, 1981; Karni \& Sagi, 1991; Ramachandran, 1976; Shiu \& Pashler, 1992). In a classification paradigm, Rentschler, Jüttner, and Caelli (1994; Jüttner \& Rentschler, 1996) found that learning the discrimination of Gabor patches differing in their third harmonic is partially specific for the visual-field position of training. In contrast to Nazir and O'Regan (1990), who found the same amount of transfer to contralateral locations as they did to the fovea, they observed a disadvantage of displacements from the periphery to the center of the visual field as opposed to shifts within the periphery. Rentschler et al. (1994) concluded that the internal representation of stimuli was fundamentally different for foveal and peripheral stimuli.

Nazir and O'Regan (1990) and Rentschler et al. (1994) had subjects learn to discriminate a certain set of patterns, whereas Foster and Kahn (1985; Kahn \& Foster, 1981) tested translation invariance without any stimulusspecific training: Their subjects had to judge whether two sequentially flashed random-dot clouds were "same" or "different." These authors found that (1) this task was easier when both clouds were presented to the same retinal location, (2) larger displacements produced larger decrements in performance, and (3) positional specificity disappeared when same stimuli were rotated in addition to translated for the second presentation. These data and a number of additional studies (reviewed in Foster, 1984,1991 ) with similar stimuli led Foster and Kahn to suggest a continuous position-shift operation to compensate for translations in the visual field. This normalization operation might require additional time to allow for a decay of "faithfulness or fidelity of the internal representation" (Foster \& Kahn, 1985).

Evidence for positional specificity in same/different recognition, however, is not unequivocal, since Phillips (1974; recently reproduced by D. E. Irwin, 1991) showed that, for interstimulus intervals (ISIs) longer than $300 \mathrm{msec}$, judgments of the identity of two checkerboards were not affected by displacement. The position shift involved in this experiment $\left(0.8^{\circ}\right.$ for stimuli with a total size of $4^{\circ}$ and more) may have been too small for the detection of any effect. On the other hand, there may also be explanations for the Foster and Kahn (1985) results that are independent of visual recognition processes, for example, the involvement of spatial attention. Given that the identification of two novel patterns as "same" or "different" is one of the simplest of recognition tests and represents a baseline for future learning studies on position invariance, we considered performance in matching tasks worth a closer look.

The main purpose of our study was to confirm positional specificity of pattern recognition and to provide evidence regarding the mechanism underlying this finding. Experiment 1 reproduced results reported by Foster and Kahn (1985) that a same/different discrimination between two successively presented dot clouds is easier if both clouds are presented to identical locations. In addition, in this experiment we examined the influence of pattern similarity on the displacement effect and tested 
the possibility that a lack of transfer might be a special problem of interhemispheric communication. In Experiments $2-4$, we controlled for the role of attentional mechanisms or eye movements. In Experiments 5 and 6, we extended the investigation to a second pattern type: black and white random checkerboards. In Experiment 5, masking stimuli were presented during the ISI in order to rule out an involvement of retinal or cortical afterimages. The employment of two different matrix sizes in this experiment also provided information on the dependency of positional specificity on stimulus complexity. Finally, Experiment 6 confirmed the effect of similarity observed in Experiment 1 and tested the influence of concurrent contrast reversal on the sensitivity to retinal translation.

\section{EXPERIMENT 1}

Many same/different studies under stimulus transformation share a common pattern of results: Changing a stimulus along one dimension, such as size or orientation, reduces performance for same trials (sometimes phrased "same performance" in the text below), while different trials can be more or less unaffected (e.g., Arnoult, 1954; Bagnara, Simion, \& Umiltà, 1984; Biederman \& Cooper, 1992; Koriat, Norman, \& Kimchi, 1991). This differential outcome has sometimes been interpreted as indicating separate processing channels for detection of "same" and "different" (reviewed in Farell, 1985).

Foster and Kahn (1985) presented their results as $d^{\prime}$, a bias-free measure of discriminability combining hit rates (i.e., the percentage of correct responses in same trials) and false-alarm rates (i.e., the percentage of errors in different trials; see Macmillan \& Creelman, 1991). A problem with Foster and Kahn's evaluation technique is that their experimental procedure involved the intermixing of two kinds of same trials in a single test: stimuli could be either displaced only or displaced and rotated by $180^{\circ}$. Different trials were not separable for the two conditions - since a different dot cloud is different from the reference regardless of orientation-and had to be included completely in the calculation of $d^{\prime}$ for either task. Foster and Kahn were aware of this problem and described their accuracy measure as " 'Same'-detection performance."

When we set out to investigate translation invariance, pilot studies (with stimuli similar to those in Experiments 5 and 6 below) confirmed the considerable decrease in performance in same trials after stimulus translation (results not shown). This decrease, however, was largely compensated for by an increase of accuracy in different trials. Hence, effects of translations on $d^{\prime}$ were small though still detectable. As our pilot studies did not involve additional rotations, we wondered whether the intermixing of two kinds of experimental conditions could have interfered with different performance in Foster and Kahn's (1985) study, leading to an overestimation of the displacement effect on $d^{\prime}$. There are several reports in the literature showing differential influences of mixed conditions in same/different experiments (e.g., Carr, Posner,
Pollatsek, \& Snyder, 1979; Krueger, 1985, 1986; Krueger \& Allen, 1987).

In order to rule out this artifactual explanation, we decided to reinvestigate, in separate sessions, the effects of displacement (horizontal session) and combined displacement/rotation (rotated session) under otherwise only slightly modified conditions: In order to stabilize fixation, we presented the fixation spot throughout each trial until immediately before the onset of a pattern. A second difference in experimental design was the negative feedback provided after incorrect answers, allowing subjects to optimally adjust their response criterion.

In addition to these two partial experiments, we also tested transfer for vertically displaced dot clouds (vertical session). With this test condition, we examined whether the effects of displacement are a special problem of information exchange between the two hemispheres of the brain. If this is the case, one would not expect to find positional specificity in the vertical task, because this does not require interhemispheric transfer.

Finally, we extended our investigation to a difficult discrimination condition involving different trials with very similar dot patterns sharing an identical configuration of five out of six dots (difficult session). The rationale for this task was that while in the other three sessions a single stimulus feature might serve as a diagnostic for discriminating same from different, the influence of such particularities should be reduced for more similar patterns. According to the suggestion by Nazir and O'Regan (1990) that invariant recognition is achieved only by detecting such features irrespective of location, one would expect positional specificity to increase in the difficult task. On the other hand, a shifting operation compensating for translations at early stages of visual processing should not be specifically affected by pattern similarity. At least simpler types of shifting models would predict differences in control and transfer performance between easy (horizontal, vertical) and difficult tasks to be proportional.

\section{Method}

Subjects. For each of the experiments reported below, 8 subjects were drawn arbitrarily from a pool of 15 (A.B., A.S., B.N., B.S., G.S., J.T., K.H., L.G., M.B., M.D., M.F., S.P., S.S., T.V., U.B.), ranging in age from 19 to 30 years (mean age 24 years). Except for one of the authors (M.D.) and 4 members of laboratory staff (A.B., J.T., L.G., T.V.), they were undergraduate students from Tübingen University and were paid for their participation. Each had normal (at least $20 / 20$ ) or corrected-to-normal visual acuity, as assessed by the Freiburg visual-acuity test (Bach, 1996). At the beginning of a session, the subjects were informed of the design of the experiment (type and locations of stimuli, presentation sequence and task) and were instructed to keep steady fixation throughout each trial. All subjects were explicitly told that their decisions on pattern identity in each trial should be independent of stimulus positions and to rely only on characteristics of the patterns themselves. It was emphasized that accuracy had to be considered as more important than speed, but that reaction times would be evaluated too. Subjects B.N., B.S., G.S., M.D., S.P., and S.S. participated in all four parts of the experiment (first horizontal, the other three in arbitrary order). They were joined by J.T. and L.G. in part horizontal, K.H. and M.B. in part vertical, and by A.S. and M.B. in part difficult and in part rotated. 
Apparatus and Stimuli. Stimuli were produced on a black-andwhite CRT monitor (refresh rate $75 \mathrm{~Hz}$ ) controlled by a Macintosh Power PC. The display was viewed binocularly at a distance of $1 \mathrm{~m}$. Stimuli consisted of 10 black dots (Figures $1 \mathrm{a}$ and $1 \mathrm{c}$ ) that were randomly distributed in a square area with an edge length of $0.5^{\circ}$. Each dot subtended a visual angle of roughly $0.035^{\circ}$. For the difficult discrimination condition, the number of dots had to be reduced to 6 in order to keep performance significantly above chance level (Figure $1 \mathrm{~b}$ ). The patterns were presented for only about $100 \mathrm{msec}$, a time too short to foveate the stimulus by a rapid saccade (Saslow, 1967). Dot clouds were displayed on a uniform white background (mean luminance, about $100 \mathrm{~cd} / \mathrm{m}^{2}$; total visual angle, $12^{\circ}$ wide $\times$ $16^{\circ}$ high). Since dot cloud presentation was terminated by renewing the white background, delayed stimulus offset due to phosphor decay can be excluded.

Fixation was aided by a black spot, about $0.13^{\circ}$ in diameter, at the middle of the monitor. Decisions were communicated by hand-held press buttons connected to the PC. Most subjects held the green "same" button in their right and the red "different" button in their left hands. A computer tone provided negative feedback immediately after incorrect responses.

Experimental design. Experiment 1 consisted of four independent parts (horizontal, vertical, difficult, rotated) performed in individual experimental sessions separated by at least 1 day. Each session comprised eight blocks of 60 trials each. The subjects initiated a block by pressing either of the two buttons. Trials in each block were balanced for identity (same vs. different), visual field (left vs. right for horizontal, difficult, and rotated, respectively; above vs. below fixation for vertical), and five displacement conditions (displacements of $0^{\circ}, 0.5^{\circ}, 1^{\circ}, 1.5^{\circ}$, and $2^{\circ}$, respectively) presented in a randomized order. During the whole session, each subject was thus tested in 48 same and 48 different trials for each displacement condition.

Individual trials in all four parts followed the same basic design: A trial started with a 1-sec appearance of the fixation spot, which was followed by the brief display of a reference stimulus, a second presentation of the fixation spot $(1 \mathrm{sec})$, and the final presentation of the test stimulus. While the reference was always centered at $1^{\circ}$ in the parafoveal visual field, the test stimulus appeared at one of five retinal locations, ranging in steps of $0.5^{\circ}$ from the position of the reference to the contralateral location. If, for example, the reference had been displayed at $1^{\circ}$ left, the test stimulus was presented at one of the following positions: $1^{\circ} \mathrm{left}, 0.5^{\circ} \mathrm{left}, 0^{\circ}$ (fovea), $0.5^{\circ} \mathrm{right}$, $1^{\circ}$ right. In principle, this design confounds degree of displacement with absolute retinal position. However, the monotonical decay of performance found by Foster and Kahn (1985) indicates that acuity effects are of minor importance and can be neglected for this task.

The ISI ( $1 \mathrm{sec}$ ) was long enough to prevent apparent-motion effects that are observed for only very short, below-300-msec ISIs

$$
\text { reference same different }
$$

$\because \because \quad \because \because \quad \because \because 8$

b)

\begin{tabular}{ccc}
$\cdot$ & $\cdot$ & $\ddots$ \\
$\cdot:$ & $\cdot$ & $\ddots$ \\
\hline
\end{tabular}

c)

$\because \because \because \quad \therefore:$

Figure 1. Dot clouds for same and different trials of Experiment 1: (a) easy task (horizontal and vertical), (b) difficult task, (c) rotated task.
(Phillips, 1974). Following the extinction of the second pattern, a question mark was displayed requesting the subject's decision of whether both stimuli were "same" or "different." Neither reference nor test patterns were visually masked. The computer generated new reference and test patterns for each trial. Two trials were separated by a 1 -sec break between the subject's response and the onset of the fixation spot.

For horizontally or vertically translated dot clouds (easy discrimination; Figure la), the test stimulus was either identical to the reference (except for a possible new position) or arbitrarily different, that is, a new random dot cloud was generated (easy discrimination). For the difficult discrimination condition, different clouds were identical in the relative arrangement of five of the six dots. Only one dot was shifted within the cloud (Figure lb), which, in addition, was horizontally displaced in $80 \%$ of the trials. For rotation, the test stimulus was either a copy of the reference rotated by $180^{\circ}$ or arbitrarily different (Figure $1 \mathrm{c}$ ).

Evaluation of the data. For each subject in this and all following experiments, the percentage of correct answers (\% correct) and the mean RT were calculated separately for each of the displacement and identity (same vs. different) conditions. RTs larger than $3 \mathrm{sec}$ were discarded prior to the calculation. Figures 2-4 and 6 represent means over all subjects. Results were statistically analyzed by two-way (main factors: translation, $\mathrm{T}$, and same/different identity, I) and one-way (main factor: T) analyses of variance (ANOVAs). Because of the artifactual influence of the fixation spot (see below), $1^{\circ}$ displacement conditions were generally excluded from the statistical analysis.

To allow for better comparison with the data published by Foster and Kahn (1985), we also determined $d^{\prime}=z$ (false-alarm rate) $-z$ (hit rate), the signal-detection measure of discriminability. For that purpose, we pooled data for each experimental condition over all subjects in order to avoid overestimation of $d^{\prime}$ on account of the limited number of trials per person (Macmillan \& Creelman, 1991; Miller, 1996). The reliability of $d^{\prime}$ values and their differences is judged by the corresponding standard errors and confidence intervals (Macmillan \& Creelman, 1991). As a control for the effects of pooling, $d^{\prime}$ values of individual subjects were calculated in all experiments and evaluated by nonparametric paired comparison (Wilcoxon signed rank). The results of this procedure are not referred to in the text because they lead to the same conclusions as for pooled data, although, in some cases, on a weaker significance level. Where two mean percentages or latencies are compared directly, significance statements refer to two-tailed, paired $t$ tests.

\section{Results}

Accuracy data for horizontal, vertical, and difficult conditions. The pattern of results for horizontal (Figures $2 \mathrm{a}$ and $2 \mathrm{e}$ ), vertical (Figures $2 \mathrm{~b}$ and $2 \mathrm{f}$ ), and difficult (Figures $2 \mathrm{c}$ and $2 \mathrm{~g}$ ) conditions is very similar: The percentage of correct responses (Figures $2 \mathrm{a}-2 \mathrm{c}$ ) in same trials decreases with increasing stimulus separation, while different trials show a slight reverse tendency. To evaluate these findings statistically, we performed two-way, repeated measures ANOVAs testing the influence of the main factors of degree of translation (T) and sameldifferent identity (I). Because of the artifactual influence of the fixation spot (see Discussion), $1^{\circ}$ displacement conditions were, as a rule, excluded from the statistical analysis. Hence, $\mathrm{T}$ comprises only four levels $\left(0^{\circ}, 0.5^{\circ}\right.$, $1.5^{\circ}$, and $2^{\circ}$ ). The analysis reveals significant influence of $T$ [horizontal, $F(3,21)=12.91, p<.001 ;$ vertical, $F(3,21)=7.31, p<.01 ;$ difficult, $F(3,21)=4.83, p<$ $.05]$, indicating that displacement increases the overall 

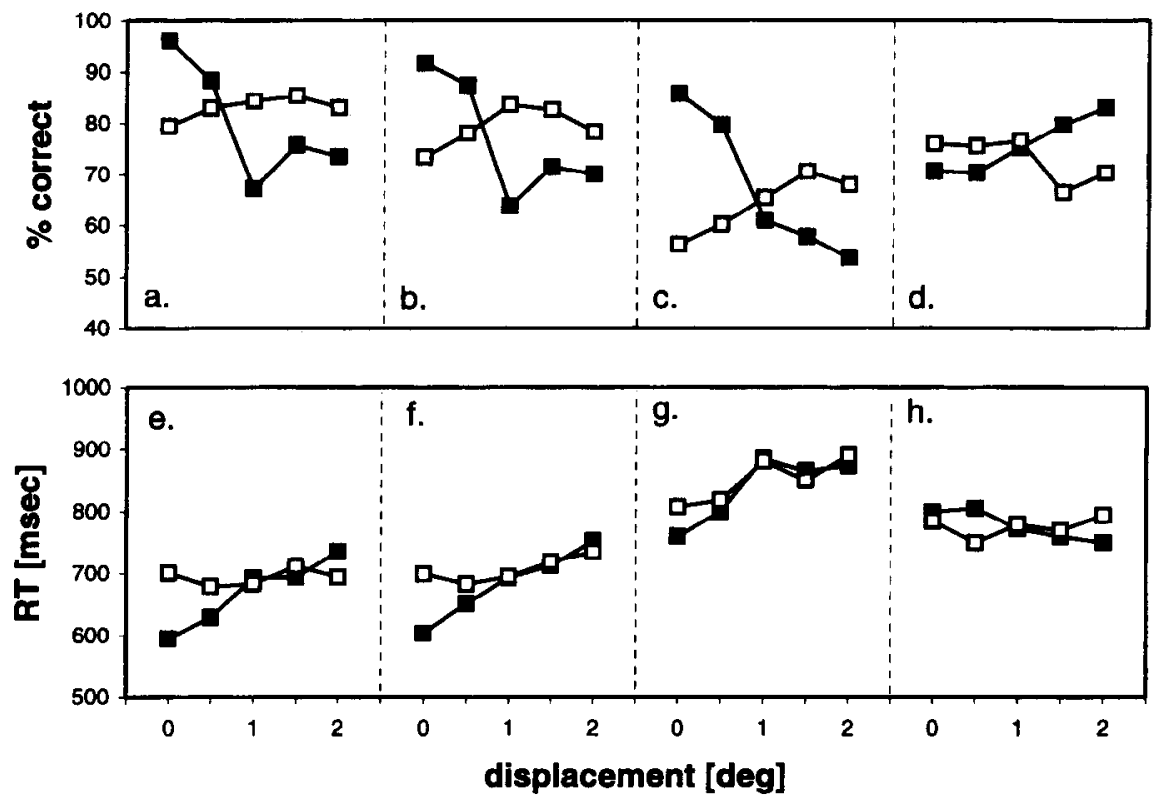

Figure 2. Experiment 1: Effects of displacement on same/different recognition of dot clouds. Mean percentage of correct responses (\% correct; panels a-d) and mean response times (RT, in milliseconds; panels e-h), calculated separately for same (filled symbols) and different (open symbols) trials. (a and e) horizontal task. (b and f) vertical task. (c and g) difficult task. (d and $h$ ) rotated task.

error frequency. This effect is largely influenced, however, by pattern identity, as evidenced by a significant interaction of $\mathrm{T} \times \mathrm{I}[$ horizontal, $F(3,21)=10.11, p<.001$; vertical, $F(3,21)=12.73, p<.001 ;$ difficult, $F(3,21)=$ $19.99, p<.001]$. The factor I, however, did not contribute significantly to performance in general [horizontal, $F(1,7)=0.02, p>.1$; vertical, $F(1,7)=0.20, p>.1$; difficult, $F(1,7)=0.60, p>.1]$, that is, there seemed to be no general preponderance of either "same" or "different" judgments. The decrease of accuracy in same trials is highly significant in one-way ANOVAs [horizontal, $F(3,21)=17.74 ;$ vertical, $F(3,21)=16.32$; difficult, $F(3,21)=20.85 ; p<.001$, respectively], while only for the difficult condition can a reliable increase in different trials be detected [horizontal, $F(3,21)=1.31, p>.1$; vertical, $F(3,21)=2.72, p<.1 ;$ difficult, $F(3,21)=$ $4.43, p<.05]$.

RT data for horizontal, vertical, and difficult conditions. RT data (Figures $2 \mathrm{e}-2 \mathrm{~h}$ ) are conclusive in that retinal shifts lead to longer latencies [horizontal, $F(3,21)=$ $10.04, p<.001$; vertical, $F(3,21)=13.31, p<.001$; difficult, $F(3,21)=12.63, p<.001]$. A speed-accuracy tradeoff, therefore, cannot be responsible for the displacement effect on error rates. Latencies are also influenced by pattern identity. This effect, however, is significant only for the two conditions with easily discriminable dot clouds [horizontal, $F(1,7)=11.36, p<.05 ;$ vertical, $F(1,7)=9.78, p<.05 ;$ difficult, $F(1,7)=2.23, p>.1]$ and interacts with displacements [horizontal, $F(3,21)=$ $14.72, p<.001$; vertical, $F(3,21)=3.30, p<.05$; diff $i$ cult, $F(3,21)=1.12, p>.1]$. This interaction, $\mathrm{T} \times \mathrm{I}$, can be described best by stating that without displacement same trials are faster [horizontal, $t(7)=5.37, p<.01$; vertical, $t(7)=2.77, p<.05 ;$ difficult $t(7)=2.32, p<.1]$, but that this fast-same effect disappears after retinal translations. In other words, displacement effects are stronger on same [horizontal, $F(3,21)=14.90, p<.001$; vertical, $F(3,21)=8.82, p<.01 ;$ difficult, $F(3,21)=$ $8.77, p<.01]$ than on different [horizontal, $F(3,21)=$ $2.10, p>.1$; vertical, $F(3,21)=3.68, p<.05$; difficult, $F(3,21)=5.19, p<.01]$ latencies.

Accuracy and RT data for the rotated condition. If same patterns are rotated in addition to being displaced (Figure $2 \mathrm{~d}$ and $2 \mathrm{~h}$ ), neither percentage correct nor RT data are significantly influenced by either of the main factors $[p s>.1$, with $F(3,21)=1.48(\%$ correct $)$ and 0.81 (RT) for factor T; $F(1,7)=0.39$ (\% correct) and $0.03(\mathrm{RT})$ for factor $\mathrm{I}]$. The interaction $\mathrm{T} \times \mathrm{I}$ contributes to variance for accuracy $[F(3,21)=4.81, p<.05]$, but not for RTs $[F(3,21)=1.94, p>.1]$. This interaction is largely attributable to a slight increase of same accuracy after displacement $[F(3,21)=4.51, p<.05]$. Foster and Kahn attributed similar findings in a variety of experiments to display-symmetry effects (Foster, 1984, 1991; Foster \& Kahn, 1985; Kahn \& Foster, 1981, 1986).

\section{Discussion}

Experiment 1 reproduces the basic findings of the Foster and Kahn (1985) study under conditions that avoided the potential artifact of intermixing two kinds of same trials: Displacement of dot clouds decreases performance; the decrease is monotonic and is not observed with ro- 
tated patterns. A comparison of $d^{\prime}$ values (Figure 7) reveals the striking similarity between our novel (horizontal, rotated) and the published (horizontal, F, rotated, F) data, showing that our manipulations of the experimental procedure (separate partial experiments, continuous fixation aid, feedback) had no decisive influence on the outcome. The new results additionally characterize the displacement effect as largely specific for same judgments, while a slight opposite tendency on accuracy is observed in different trials. We had expected differential consequences of displacement for same and different trials from our pilot studies. However, this finding does not explain the displacement effect: The improvement of different performance with displacement is small compared with the decrease of same accuracy and reaches significance only for the difficult condition.

For RT data, the significant interaction $\mathrm{T} \times \mathrm{I}$ is basically due to a fast-same effect vanishing after translation. The phenomenon that responses are faster to same than to different patterns is frequently encountered in matching tasks. Much research has been done on this topic in the past three decades without resolving the debate on the underlying mechanisms completely (see, e.g., Farell, 1985; Krueger, 1978; Proctor, 1983). It is worth noting that despite many investigations on the influence of stimulus position in the visual field (e.g., J. R. Irwin \& Francis, 1995; Krueger, 1985; Schweitzer, 1991) on the fast-same effect, sensitivity to retinal translations (i.e., location changes) has not been studied in any detail (but see Chignell \& Krueger, 1984; Kwak, Dagenbach, \& Egeth, 1991). Our experiments were not designed to enter this field of research, yet they indicate some positional specificity of the fast-same effect.

The pattern of results for horizontal and vertical translations is largely identical. The displacement effect is therefore not a special property of interhemispheric information transfer. It is, rather, a more general phenomenon, as confirmed by translation-variant performance under difficult discrimination conditions: Increasing the similarity of the patterns clearly reduces overall performance as compared with the two tasks involving easily discriminated patterns (from $87.7 \%$ for horizontal to $71.0 \%$ for difficult, for $0^{\circ}$ displacement each), but does not interfere with the size of the displacement effect. Mean accuracy drops after $2^{\circ}$ shifts by $10.0 \% \pm 2.1 \%$ in the horizontal, $8.0 \% \pm 2.6 \%$ in the vertical, and $10.2 \% \pm$ $1.84 \%$ in the difficult tasks. The similarity of the dot clouds thus seems to exert influence on position-invariant components of recognition, though not on positionspecific processes (see also Figure 7). Interestingly, Edelman (1995) found that invariance for 3-D rotations was also less pronounced for more similar objects. Further studies are needed for a decision on whether or not both corresponding results represent a general relation of similarity and invariance.

"Same" recognition is especially affected by $1^{\circ}$ displacements. Since, at that location, the fixation spot is removed immediately before the onset of the test pattern, the dip in the otherwise monotonically decaying curves is most probably an artifact due to a forward-masking effect exerted by the fixation spot. We were aware of this possible interference from the beginning, but did not change the experimental procedure, because we wanted the fixation of our subjects to be guided as long as possible before stimulus onset. Furthermore, this artifactual part of the experiment provided an indirect fixation control: At least at the onset of the test pattern, subjects were not looking around, otherwise the influence of the artifact would be smeared over a larger position range. Nevertheless, for the above ANOVAs, percentages and response times at $1^{\circ}$ displacement were discarded and, therefore, could not influence the interpretation of the displacement effect. For all other experiments presented below, we avoided this problem by presenting reference and test patterns only in the parafovea.

A somewhat puzzling finding is that this fixation artifact exerts no influence (or only a minor one in Figures $2 \mathrm{~b}$ and $2 \mathrm{~g}$ ) on different recognition. While this finding at first sight seems to support the notion of two separate recognition processes for same and different, it remains unclear as to how an independent different detector would be able to escape the forward-masking influence of the fixation spot. This new same/different disparity may be better understood by assuming that different decisions rely on the absence of evidence for same (see General Discussion).

\section{EXPERIMENTS 2-4}

When observing positional specificity in a visual task, it is tempting to attribute this effect to eye movements or to spatial attention: The presentation of the reference stimulus might induce a saccade or a shift of the focus of attention, thereby increasing visibility at control positions and decreasing performance for translated patterns. In Experiment 1, the fixation spot was displayed throughout each experimental trial and subjects were instructed to carefully keep steady fixation. The presentation time of 100 msec was short enough to keep subjects from performing foveating saccades after stimulus onset (Saslow, 1967). These experimental conditions make the involvement of saccades rather improbable. That the displacement effect in Experiment 1 is largely specific for same trials and disappears for rotated stimuli not only corroborates the absence of eye-movement artifacts but simultaneously provides first evidence against an unspecific role of attention. To rule out contributions of any recognition-independent but position-directed effects, we designed three short control experiments.

In Experiment 2, shifts of gaze or attention were excluded by the brief simultaneous display of two patterns. Kahn and Foster (1981, 1986; see also Foster, 1984) had already presented control experiments indicating that the displacement effect could be found even with simultaneously presented stimuli. Since it is not possible to show two individual patterns at the same time and place, these 
data contain no control for $0^{\circ}$ displacement. To be able to compare a displacement condition with a suitable zero control, we chose the following design: The short presentation of two arbitrarily different dot clouds $1^{\circ}$ to the left and right of the fixation spot was followed by a single test stimulus at one of the two locations. Subjects had to judge whether or not the test pattern was identical to either of the two reference stimuli.

Experiments 3 and 4 replicated the critical part $\left(0^{\circ}\right.$ and $2^{\circ}$ displacements) of the horizontal task of Experiment 1 with some modification of the procedure: In Experiment 3 , subjects knew in advance where the test stimulus would appear relative to the reference pattern, because performance with and without displacement was tested in separate blocks. In Experiment 4, an additional different dot cloud cued the position of the test stimulus. Under these conditions, voluntary as well as reflex-like eye movements or shifts of the attentional spotlight should be equally likely for control and transfer positions. Both modifications of the standard procedure should also help to elucidate the role of the interactions of displacement and pattern identity.

\section{Method}

Subjects. The following subjects were tested in the three experiments ( $n=8$, respectively): A.S., B.N., B.S., G.S., M.B., M.D., S.P., and S.S. in Experiment 2; A.B., B.N., K.H., M.D., M.F., S.P., S.S., and U.B. in Experiment 3; and A.B., A.S., B.N., M.D., M.F., S.P., S.S., and T.V. in Experiment 4. Most of the subjects had already taken part in Experiment 1 or in other psychophysical experiments on translation invariance.
Apparatus and Stimuli. The experimental setup was the same as in Experiment 1. Ten-dot stimuli, as in Figure 1a, were presented at $1^{\circ}$ eccentricity to the left and right of the fixation spot.

Experimental design. Each trial of Experiment 2 started with a 1 -sec display of the fixation spot followed by two arbitrarily different dot clouds presented simultaneously at $1^{\circ}$ to the left and right of the fixation spot. After a $1-\mathrm{sec}$ interstimulus interval, a single test stimulus was shown at one of the two locations. Subjects were to judge whether the test pattern was identical to either one of the two reference stimuli ("same") or not ("different"). The experiment was performed in four blocks of 64 trials each. In 32 of these trials, the test pattern was different from the two reference stimuli; in 16 trials, the test pattern corresponded to the reference at the same and at the contralateral positions, respectively. As in all other experiments presented here, the order of the trials was randomized within one block.

Both Experiments 3 and 4 replicated the $0^{\circ}$ (control) and $2^{\circ}$ horizontal displacement conditions of Experiment 1. In Experiment 3, control and displacement trials (256 each) were separated in two consecutive parts consisting of four blocks each. Half of the subjects passed the control part first, the other half began with the displacement trials. The two parts were separated by a break of at least $5 \mathrm{~min}$.

In Experiment 4, control and displacement trials were again mixed in each of five blocks ( 64 trials per block), but an additional, different dot cloud appeared $1 \mathrm{sec}$ after presentation of the reference cloud for $300 \mathrm{msec}$ at the same position at which the test stimulus was to be displayed another $1 \mathrm{sec}$ later. Hence, reference and test stimuli were separated by a total ISI of $2.3 \mathrm{sec}$. The purpose of the intervening cloud was that of a cue to the location of the test pattern.

\section{Results}

Consistent with the result in Experiment 1, correct responses in Experiment 2 were more frequent [Figure 3a;
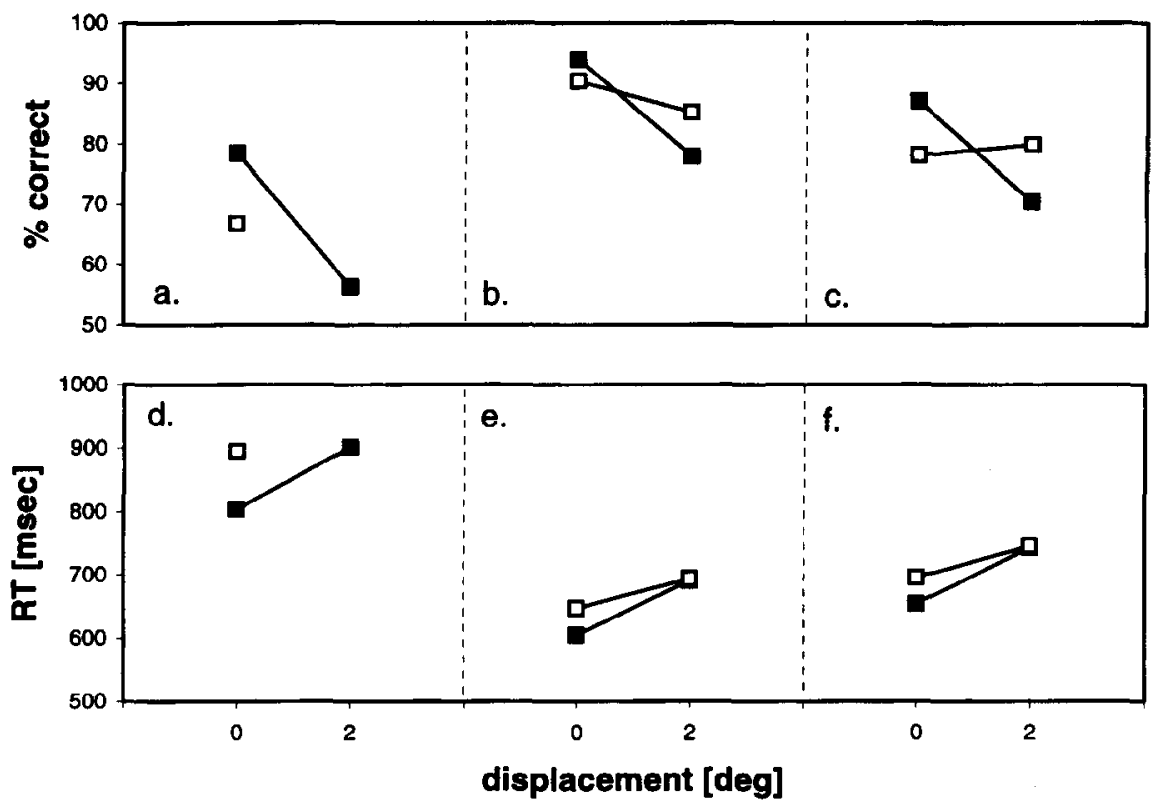

Figure 3. Experiments 2-4: Mean percentage of correct responses (panels a-c) and mean response times (d-f). Results are shown separately for same (filled symbols) and different (open symbols) trials. (a and d) Experiment 2: Two simultaneous reference patterns. Results of different trials cannot be separated into control and displacement conditions and are represented in the figure by a single open symbol shown at $0^{\circ}$. (b and e) Experiment 3: Effects of blocked testing. (c and $f$ ) Experiment 4: Effects of position cuing. 
$t(7)=5.28, p<.01]$ and faster [Figure $3 \mathrm{~d} ; t(7)=2.39$, $p<.05$ ] for test patterns that corresponded to reference stimuli at the same retinal position than they were for test patterns that were identical to the dot cloud from the alternative location. This effect cannot be attributed to general or individual asymmetries of pattern recognition (say that a subject pays attention only to the left visual hemifield and therefore recognizes only test stimuli presented there): Differences in accuracy between trials involving test patterns in the left and right visual fields are negligible (mean absolute difference $1.56 \% \pm 0.46 \%$ ) as compared with the differences between the control and displaced same conditions $(22.26 \% \pm 4.22 \%)$.

The results of Experiments 3 and 4 are also depicted in Figure 3 (middle and right panels, respectively). In both cases, accuracy and response times were influenced by retinal displacements [Experiment 3, \% correct, $F(1,7)=78.73, p<.001 ;$ RT, $F(1,7)=18.34, p<.01$; Experiment $4, \%$ correct, $F(1,7)=37.89, p<.001$; RT, $F(1,7)=16.02, p<.01]$, while pattern identity did not contribute significantly to variance $[p>.1$, with $F \mathbf{s}(1,7)=$ $0.37,0.83,0.01$, and 1.12 , respectively]. The interaction of both factors is evident for accuracy [Experiment 3, $F(1,7)=11.97, p<.05$; Experiment $4, F(1,7)=20.02$, $p<.01$ ], but less so for RT [Experiment 3, $F(1,7)=$ $1.33, p>.1$; Experiment $4, F(1,7)=4.38, p<.1$ ]. A fastsame effect at $0^{\circ}$ displacements is present in both Experiment 3 and Experiment 4, yet is significant only in the latter $[t(7)=2.54, p<.05$; in Experiment 3, $t(7)=$ $1.11, p>.1]$. In both experiments, effects of displacement on same accuracy and latency are all significant at $p<$ .01 and are stronger than the influence on different trials that are reliable for accuracy only in Experiment $3[t(7)=$ $3.03, p<.05]$ and for latency only in Experiment $4[t(7)=$ $3.15, p<.05]$. Overall performance was slightly reduced in Experiment 4 as compared with that in Experiments 1 and 3. Given the results of Experiment 5 (see below), this is a consequence of the increased ISI between reference and test pattern rather than of a masking influence of the position cue.

\section{Discussion}

An appealing explanation for the displacement effect would be that the presentation of the reference attracts spatial attention or evokes rapid saccades to that location, thereby yielding better performance for control than for displacement trials. Actually, a comparison of performance levels in Experiment 2 with "standard" samel different experiments corroborates the importance of spatial attention: Perceiving two patterns simultaneously increases error rates and latencies in both same and different trials. Sharing attention between two patterns at a time evidently makes the task more difficult. The displacement effect, however, seems to be insensitive to attentional demands: Neither manipulations allowing the direction of all resources to the test location (Experiments 3 and 4) nor those impeding it (Experiment 2) exert any influence on positional specificity. All three controls clearly show sensitivity of recognition performance to translations consistent with the results of Experiment 1. Attentional shifts as well as eye-movement artifacts can, therefore, be ruled out. Any argument of increased or reduced visibility because of auxiliary mechanisms independent of visual pattern recognition and/or memory would be insufficient to explain position variance.

Besides serving as a control for eye movements or attentional shifts, Experiment 3 also tested for a possible influence of intermixing different levels of displacement in Experiment 1. Previous studies (e.g., Krueger, 1985, 1986) have shown that testing various experimental conditions in a single block yields differential effects on same and different trials similar to those we observed in Experiment 1 . These may be accounted for either by a single compromise criterion's being chosen for all trials by the subjects or by inertia of criterion readjustment between two sequential trials. That testing in separate blocks for control and displacement leads to a significant decrease in different performance (Figure 3b)-not an increase as in Experiment 1 (Figures 2a, 2b, and 2c) indicates that criterion problems do, indeed, influence response behavior in intermixed sessions. However, they are not responsible for the displacement effect, because the latter is not reduced by blocking (compare, e.g., horizontal and Experiment 3 in Figure 7). For this reason, and since blocking has the disadvantage that variance might increase on account of serial effects (e.g., fatigue or learning; see also below), we continued to employ intermixed sessions.

It is interesting to note that same trials in Experiment 3 are still more strongly affected by retinal translations than are different trials (cf. interactions $\mathrm{T} \times \mathrm{I}$ ). Other than in the published studies, where interactions of experimental condition and same/different identity disappeared in blocked tests, our subjects obviously still used a compromise criterion. A post hoc analysis of the data offers a possible explanation for this finding: The differential effects in Experiment 3 (Figures $3 \mathrm{~b}$ and $3 \mathrm{e}$ ) stem largely from performance in the first half of the session $[F(1,3)=$ $11.52, p<.05 ; 4$ subjects for control and displaced, respectively] while they are virtually absent in the second part $[F(1,3)=0.12, p>.1]$. Since all subjects had participated in intermixed experiments before (as, e.g., in Experiment 1), they may have applied, at the beginning of Experiment 3, a response strategy acquired in earlier sessions. Further experiments will be needed to test this hypothesis.

The fast-same effect and its disappearance following retinal translations found in Experiment 1 are confirmed statistically in Experiment 4 and also seem to tend to be present in Experiment 3 (Figure 3e). RT data of Experiment 2 corroborate the conclusion that the fast-same effect depends on retinal location: same control trials are nearly $100 \mathrm{msec}$ faster than different trials $[t(7)=2.41, p<.05]$, while same displaced trials are not $[t(7)=0.23, p>.1]$. 


\section{EXPERIMENT 5}

All experiments reported above confirm positional specificity for the recognition of dot clouds. Unpublished pilot studies with a different pattern type, however, yielded much smaller displacement effects, an observation to be confirmed by Experiments 5 and 6 . The stimulus type was a checkerboard matrix containing a random order of black and white elements. Size and left-right symmetry of the patterns and eccentricity and duration of their presentation were adapted from Nazir and O'Regan's (1990) work. There was no special purpose in these manipulations except for increasing the range of experimental conditions for the displacement effect. Patterns in different trials differed by just two symmetric random elements changed from black to white or vice versa.

In Experiment 5, this second pattern type was used to test the influence of the simple persistence of an unprocessed visual input at retinal, geniculate, or early cortical levels. Phillips (1974) reports a retinotopic memory store accounting for positional specificity in samel different tasks at interstimulus intervals below $300 \mathrm{msec}$. Although the ISI in our experiments was considerably longer (1 sec in Experiments 1-3, $2.3 \mathrm{sec}$ in Experiment 4; in an unpublished experiment we increased the ISI even up to $4 \mathrm{sec}$ without any obvious reduction of the displacement effect), we decided to control for possible longer lasting forms of visual persistence by inserting a pattern mask $350 \mathrm{msec}$ after the offset of the reference matrix.

As a second factor, we manipulated checkerboard complexity with half of the trials involving $3 \times 6$ and $6 \times 6$ elements, respectively. Thus, four conditions were tested in Experiment 5: $3 \times 6$ unmasked, $3 \times 6$ masked, $6 \times 6$ unmasked, and $6 \times 6$ masked.

\section{Method}

Subjects. Subjects B.N., B.S., G.S., K.H., M.B., M.D., S.P., and S.S. took part in Experiment 5.

Apparatus and Stimuli. Random matrices ("checkerboards") contained six columns and either three or six rows of elements $(3 \times 6$ and $6 \times 6$ matrices), which, in each stimulus, were randomly set to either black (about $1 \mathrm{~cd} / \mathrm{m}^{2}$ ) or white (about $100 \mathrm{~cd} / \mathrm{m}^{2}$ ) on a white background. The masking checkerboard was nonrandom in that black and white elements alternated in horizontal and vertical directions (except at the midline to allow for mirror symmetry). The $6 \times 6$ patterns subtended about $0.85^{\circ}$ in width and height. For the $3 \times 6$ checkerboards, the lower half was cut off and set to background brightness. All checkerboards were mirror symmetric relative to their vertical axes. They were presented with their centers at an eccentricity of $2.4^{\circ}$ left or right from the fixation spot. Presentation time was 150 msec.

Experimental design. Each subject performed a total of 640 trials in 10 blocks of 64 trials each, balanced for same versus different identity, $3 \times 6$ versus $6 \times 6$ patterns, masked versus unmasked, control versus displaced, and left versus right visual field. Each trial started with a 1 -sec presentation of the fixation spot, followed by the $150-\mathrm{msec}$ reference display. In all trials, the ISI before the onset of the test matrix was $1,650 \mathrm{msec}$. In half of the trials, two identical 300-msec masks were presented at both left and right locations $350 \mathrm{msec}$ after the reference had disappeared. The test pattern was either identical to the reference (same) or differed from it in two symmetrical elements changed from black to white or from white to black (different). Formally, the sequence of events in masked trials is very similar to that of Experiment 4 , the position cue potentially also having masking effects. The cue, however, was presented with a longer onset asynchrony and only at one location.

\section{Results}

Positional specificity is observed for accuracy (Figures 4a-4d) in all four parts of Experiment 5 [ $3 \times 6$ unmasked, $F(1,7)=5.81, p<.05 ; 3 \times 6$ masked, $F(1,7)=40.11$, $p<.001 ; 6 \times 6$ unmasked, $F(1,7)=10.29, p<.05 ; 6 \times 6$ masked, $F(1,7)=8.05, p<.05]$, accompanied by an interaction $\mathrm{T} \times \mathrm{I}[3 \times 6$ unmasked, $F(1,7)=3.98, p<.1$; $3 \times 6$ masked, $F(1,7)=25.55, p<.001 ; 6 \times 6$ unmasked, $F(1,7)=17.41, p<.01 ; 6 \times 6$ masked, $F(1,7)=$ $33.99, p<.001]$. As in all the above experiments, accuracy in same trials is strongly affected by displacement, while different performance in three of the four conditions (Figures $4 b-4 d$ ) shows a slight opposite tendency that is significant only for $3 \times 6$ masked $[t(7)=2.9$, $p<.05]$. Identity contributes significantly only to $6 \times 6$ masked performance $[F(1,7)=11.65, p<.05]$. Hence, accuracy data with this second pattern type is qualitatively similar to those of Experiments 1-4, although the precise pattern of results for same and different trials is less homogeneous. The mixing of conditions in one single experiment may be responsible for the greater variability of effects.

For response times (Figures $4 \mathrm{e}-4 \mathrm{~h}$ ), the positional specificity is even less pronounced - especially for the smaller matrix size $[3 \times 6$ unmasked, $F(1,7)=2.55, p>.1 ; 3 \times 6$ masked, $F(1,7)=1.06, p>.1]$ and reaches significance only for the $6 \times 6$ masked condition $[F(1,7)=9.67, p<.05$; the $F$ value for the $6 \times 6$ unmasked condition is $3.31, p>$ .1]. Except for an effect of identity in the $3 \times 6$ unmasked condition $[F(1,7)=6.05, p<.05]$ and an interaction $\mathrm{T} \times \mathrm{I}$ for $3 \times 6$ masked condition $[F(1,7)=6.37, p<.05]$, no other significant contributions were detected.

Insertion of the mask did not influence the results in Experiment 5. Two-way repeated measures ANOVAs for accuracy as well as for response time data did not reveal any significant interaction of the two main factors, translation and mask [\% correct, $F(1,7)=0.16, p>.1$; RT, $F(1,7)=0.67, p>.1]$ or any noticeable contribution of mask alone [\% correct, $F(1,7)=1.20, p>.1 ; \mathrm{RT}$, $F(1,7)=0.36, p>.1]$. In ANOVAs testing the influence of translation and complexity, that is, the number of pattern elements, the factor complexity had strong effects $[\%$ correct, $F(1,7)=83.58, p<.001 ; \mathrm{RT}, F(1,7)=73.03$, $p<.001]$, but did not interact with the positional specificity $[\%$ correct, $F(1,7)=0.36, p>.1 ; \mathrm{RT}, F(1,7)=$ $2.26, p>.1]$. No higher order interactions between complexity, mask, and translation were detected by threeway ANOVAs (all $p \mathrm{~s}>.1$ ).

\section{Discussion}

While, in Experiments 1-4, the maximal displacement was $2^{\circ}$, patterns in Experiment 5 were shifted by $4.8^{\circ}$. One would expect, therefore, a displacement effect at least as large as that in the dot-cloud experiments. Recog- 

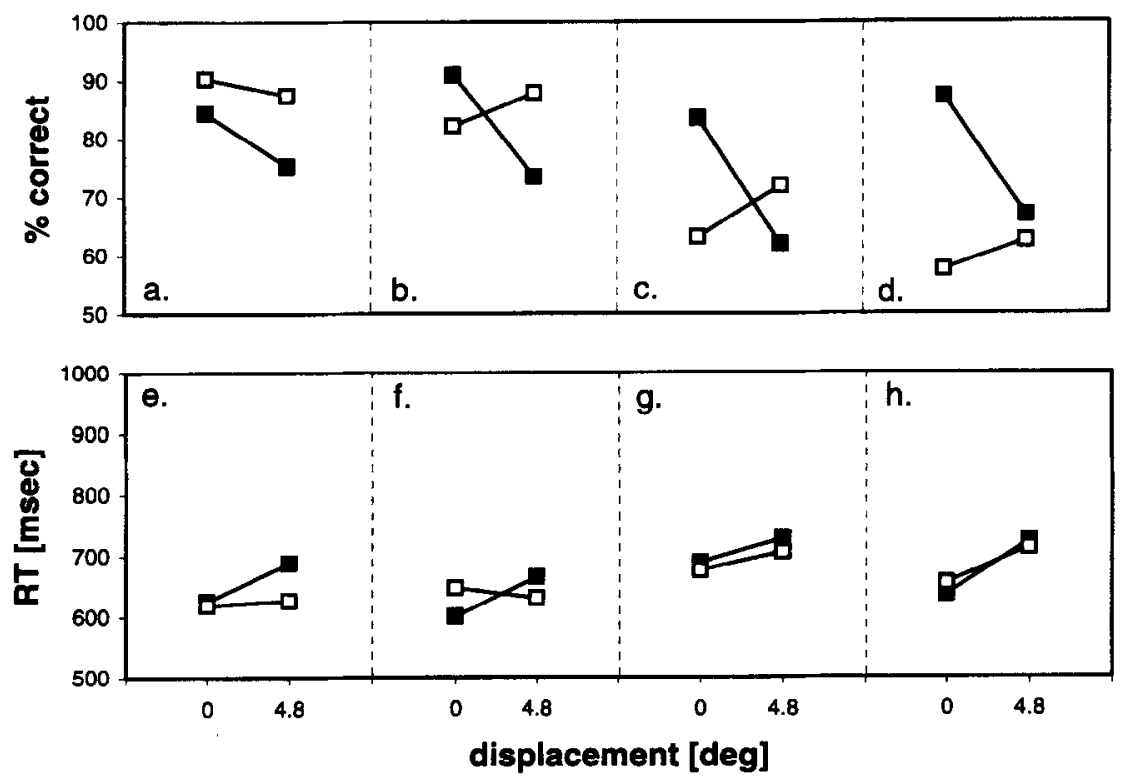

Figure 4. Experiment 5: Influences of pattern complexity and masking on the displacement effect for random checkerboards. Mean percentage of correct responses (a-d) and mean response times (e-h) for same (filled symbols) and different (open symbols) trials. (a and e) $3 \times 6$ unmasked trials. (b and f) $3 \times 6$ masked trials. (c and g) $6 \times 6$ unmasked trials. (d and h) $6 \times 6$ masked trials.

nition of checkerboard patterns actually proved to be sensitive to retinal translations. For RTs, however, the displacement effect hardly reaches significance, and for accuracy, it is at least not larger, in fact is even slightly smaller, than it is for dot stimuli, despite the larger shift of location. This will be more obvious in Experiment 6 .

Presenting a mask during the ISI turned out not to have any significant influence either on the absolute performance level or on the displacement effect (Figures 4 and 7). This holds true for matrices of both complexity levels and indicates that simple persistence of the unprocessed visual input does not contribute to position-variant results in our same/different tasks. This is consistent with all previous research on visual integration effects in showing that masking can interfere with recognition only in a very narrow time window, one of less than $300 \mathrm{msec}$ following the test stimulus. The robustness of the displacement effect suggests that translation-sensitive performance relies on processes elaborate enough to clearly discriminate between the test stimulus and the quite similar mask.

The second factor we tested in this experiment, pattern complexity-as defined by the number of checkerboard elements-also remains without a detectable influence on positional specificity, although sameldifferent judgments are clearly more difficult for the more complex $6 \times 6$ matrices. This result is reminiscent of the finding of Experiment 1 that increasing the similarity of dot clouds reduces overall performance but leaves the displacement effect largely unchanged. It should be noted that both observations may be directly related, because the proportion of identical elements is larger in different $6 \times 6$ $(34 / 36$ elements $=94 \%)$ than in different $3 \times 6$ patterns
$(16 / 18$ elements $=89 \%)$. The smaller matrices may therefore also be considered as less similar to each other. In Experiment 6, we took a closer look at the influence of pattern similarity for patterns of equal size.

\section{EXPERIMENT 6}

In both Experiment 1 and Experiment 5 easier (horizontal and vertical in Experiment 1;3 36 patterns in Experiment 5) and more difficult discrimination conditions (difficult in Experiment 1 and $6 \times 6$ patterns in Experiment 5) yielded displacement effects that were comparable in size despite clear differences in overall performance (Figures 2 and 4; see also Figure 7). Similarity of the patterns - defined as the proportion of elements identical in two different stimuli - may be more detrimental to position-invariant than to translation-sensitive components of recognition performance.

One may object, however, that element number may also have had some influence on the above findings, since, in Experiment 5, similarity was varied by means of the matrix size and, in Experiment 1, dot number in the difficult task was lowered to 6 to allow observers to achieve more than chance performance. We decided to retest the role of similarity in an experiment with checkerboards of equal dimensionality and, thus, of identical element number. In one part (easy; Figure $5 b$ ) of Experiment 6 , pattern differences in different trials were arbitrary in that each element of the test matrix was set randomly to black or white, irrespective of the corresponding element of the reference. In the second part (difficult; Figure 5a), different test stimuli were identical 


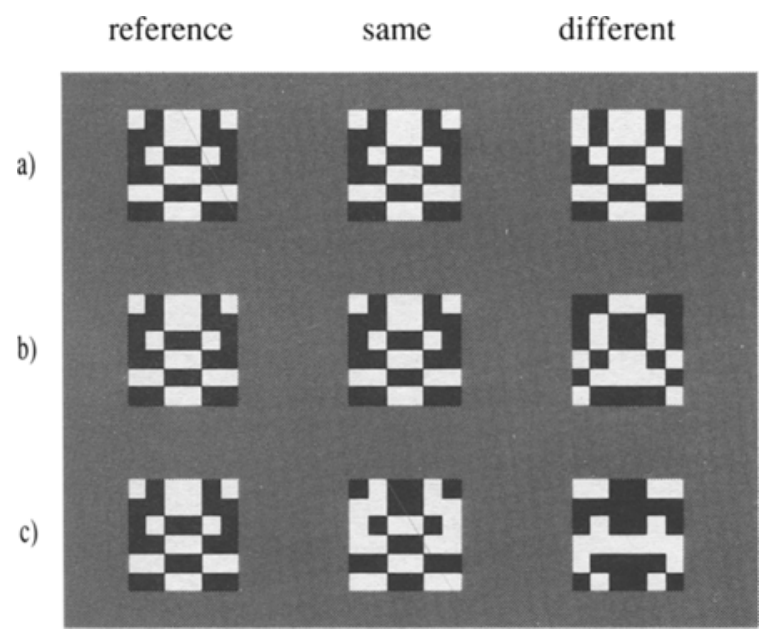

Figure 5. Checkerboard stimuli for Experiment 6. (a) Difficult task, (b) easy task, and (c) contrast-reversal task.

to $\mathrm{R}$ except for one element and its symmetrical counterpart (cf. Experiment 5).

The third part of Experiment 6 (contrast reversal; Figure $5 \mathrm{c}$ ) was designed to test another assumption: Given the above result (Experiment 1) that recognition of rotated patterns is translation invariant, one might expect that transformations other than rotation would remove positional specificity as well. We therefore probed the effects of contrast reversal in addition to displacement of a checkerboard.

\section{Method}

Subjects. Eight subjects (A.B., A.S., B.S., K.H., M.D., S.P., S.S., and $U . B$.) were tested in the three parts (easy, difficult, and contrast reversal) of the experiment.

Apparatus and Stimuli. Black and white $6 \times 6$ checkerboard stimuli, like those in Experiment 5, were presented on a now-darkgray background $\left(30 \mathrm{~cd} / \mathrm{m}^{2}\right)$. Different patterns were completely unrelated in the easy part of the experiment, in that each element of the test matrix was set randomly to black or white, irrespective of the corresponding element of the reference (for an example, see Figure $5 \mathrm{~b}$ ). In the difficult task, different patterns were identical except for two symmetrical elements (Figure 5a). In the contrastreversal task, a different test pattern was arbitrarily different from the reference, while a same test pattern was the negative of the reference, that is, each element changed its brightness from black to white or vice versa (Figure 5c).

Experimental design. Each part of the experiment consisted of 320 trials, tested in five blocks each balanced for visual field position, displacement condition, and identity. One second after the appearance of the fixation spot, the reference was displayed for $150 \mathrm{msec}$, followed by a 1 -sec ISI and the 150 -msec test pattern.

\section{Results and Discussion}

Accuracy data from Experiment 6 reveal only minor influences of factor $\mathrm{T}$ for both easy [Figure $6 \mathrm{a}, F(1,7)=$ $3.09, p>.1]$ and difficult discrimination tasks [Figure $6 \mathrm{~b}, F(1,7)=1.35, p>.1$, but a more prominent impact of the interaction $\mathrm{T} \times \mathrm{I}$ [easy, $F(1,7)=11.44, p<$ .05 ; difficult, $F(1,7)=14.44, p<.01]$ and, in the difficult task, of pattern identity $[F(1,7)=14.82, p<.01]$. The displacement effect becomes obvious and significant only when comparing $d^{\prime}$ values (Figure 7): Easy performance decreases from $d^{\prime}=3.03 \pm 0.11$ (pooled $d^{\prime} \pm$ standard error; see Method section of Experiment 1) for control to $d^{\prime}=2.68 \pm 0.10$ for displaced conditions. Difficult accuracy drops from $d^{\prime}=1.36 \pm 0.08$ to $d^{\prime}=1.08 \pm 0.07$. The difference $\Delta$ between control and displaced values is comparable in size between the two tasks (easy, $\Delta=$ $0.35 \pm 0.15$; difficult, $\Delta=0.28 \pm 0.11$ ), even though the absolute level of discriminability at the control location is quite different $(p<.001)$. Hence, Experiment 6 confirms the finding of Experiments 1 and 5 that positionspecific components of recognition are less sensitive to changes in pattern similarity than are translation-invariant contributions. It further rules out an effect of element number that could have influenced the former results.

The displacement effect is more obvious in RT (Figures $6 \mathrm{~d}$ and $6 \mathrm{e}$ ) than in accuracy data. Latencies increase significantly with translations [easy, $F(1,7)=6.48, p<$ .05 ; difficult, $F(1,7)=45.04, p<.001]$, an effect that interacts with same/different identity [easy, $F(1,7)=25.45$, $p<.01$; difficult, $F(1,7)=7.01, p<.05$ ]. In both Experiment 5 and Experiment 6 , therefore, subjects did not manage to achieve complete translation invariance of same/ different recognition. Displacement effects, however, are weaker than for dot clouds (compare, e.g., Experiments 1 and 6 in Figure 7) and may be found with either RTs or error rates but not with both to the same extent. The reasons why checkerboards yield a smaller displacement effect are largely obscure: One may speculate that checkerboards are much more familiar than dot clouds or that pattern symmetry might have an influence. We are currently investigating the latter hypothesis.

As in the above experiments, displacement affects same and different trials differentially: Influences on same accuracy and latency are obvious in both easy and difficult conditions ( $p<.01$, respectively). In different trials with difficult patterns, a significant increase in accuracy $[t(7)=2.52, p<.05]$ is again accompanied by longer latencies $[t(7)=4.10, p<.01]$.

After contrast reversal, no significant displacement effect for accuracy (Figure 6c) or RTs (Figure 6f) is observed $[F(1,7)=0.03$ (\% correct) and 0.13 (RTs), $\Delta=$ $0.03 \pm 0.11, p s>.1$, respectively), and neither does the influence of identity reach significance $[F(1,7)=0.32$ (\% correct) and 2.24 (RTs), $p \mathrm{~s}>.1]$. Hence, contrast reversal of same patterns removed positional specificity, as did rotation in Experiment 1 (Figure 2d). Interestingly, the interaction $T \times I$, which is prominent in both easy and difficult conditions, is only marginal after contrast reversal $[F(1,7)=1.90(\%$ correct $)$ and $1.36(\mathrm{RTs}), p \mathrm{~s}>$ $.1]$, indicating that the displacement effect and its interaction with same/different judgments rely on the same source.

\section{GENERAL DISCUSSION}

\section{Position Invariance via Normalization?}

Our above results show that same/different judgments for novel visual patterns are partially specific for retinal 

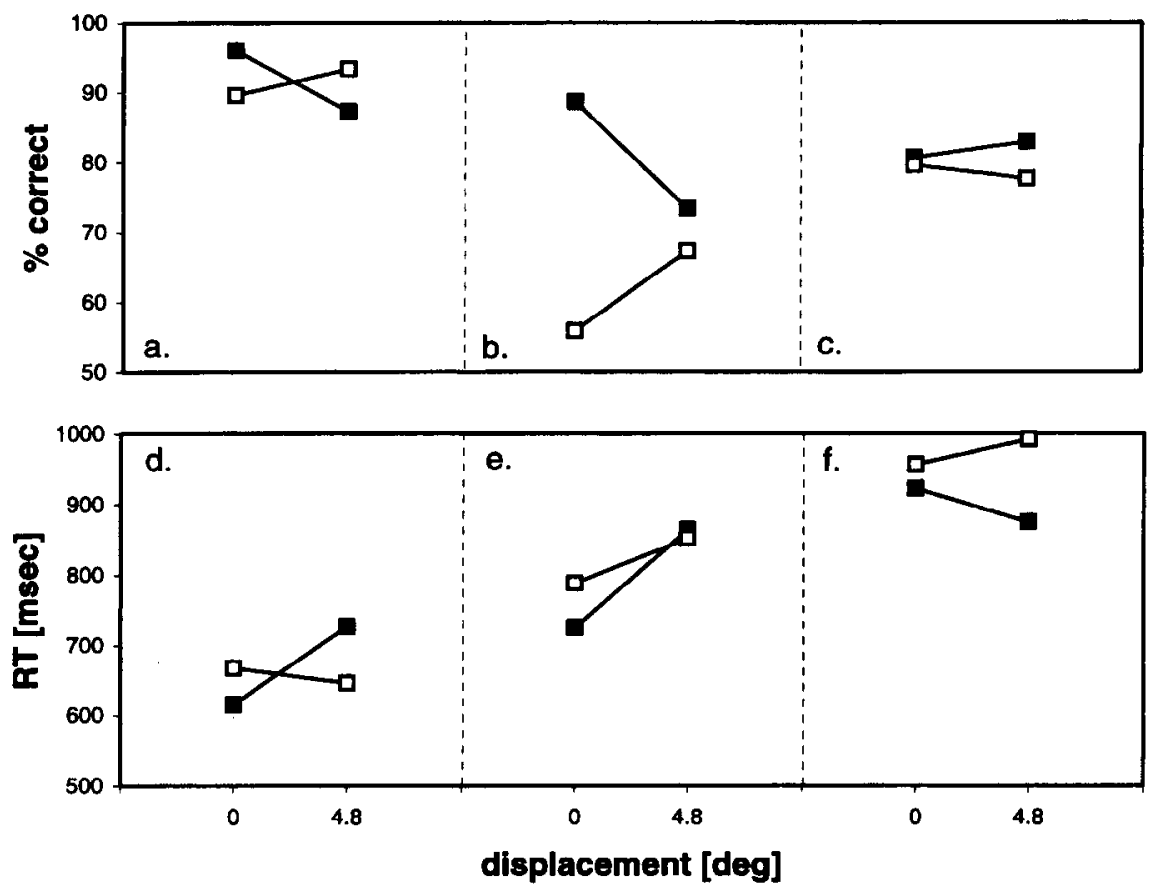

Figure 6. Experiment 6: Influences of pattern similarity and contrast reversal on the displacement effect for random checkerboards. Mean percentage of correct responses $(a-c)$ and mean response times (d-f) for same (filled symbols) and different (open symbols) trials. (a and d) Easy task. (b and e) Difficult task. (c and $f$ ) Contrast-reversal task.

location. Translation variance is observed for horizontal and vertical displacements, for easier and more difficult discrimination tasks, and for two different pattern types (dot clouds and checkerboards). The displacement effect vanishes if stimuli are rotated or contrast reversed in addition to being displaced. Positional specificity cannot be attributed to eye movements, spatial attention, or afterimages. Finally, in basically all experiments - except for those with additionally transformed stimuli where both effects are absent - positional specificity of recognition (factor T) and a differential influence on same and different trials (interaction $\mathrm{T} \times \mathrm{I}$ ) coincide. Is there a simple model for translation invariance explaining all this evidence for translation variance?

Foster and Kahn (1985) discuss a mental-shift operation aligning the two patterns to be compared. This normalization may cost time and allow for some memory decay, leading to an increase in error rates. While a mental-shift explanation of this specific type can readily account for the monotonic rise of RTs and error rates with increasing displacements, there are some objections to this view based on inconsistencies in the RT data: First, RTs are influenced by factors other than displacement, for example, task difficulty: Even without any displacement, latencies are at least $100 \mathrm{msec}$ longer under difficult than under easy conditions. Displacements may affect RTs because they increase task difficulty, the required delay being the consequence of, and not the reason for, positionvariant recognition. Second, in several of our experiments (see, e.g., Figures $2 e$ and 2f) latencies for different trials do not vary with position, while RTs in same trials clearly do. The speed of the mental transfer mechanism therefore would depend on the result of a process supposed to be based on the transfer! In other experiments (see, e.g., Figures $2 \mathrm{~g}, 4 \mathrm{~g}, 4 \mathrm{~h}$, and $6 \mathrm{e}$ ), RTs in different trials increase with translation, whereas error rates decrease. Taken together, these results show that changes of RTs after pattern displacement are neither necessary nor sufficient for an increase in error rates. Only a number of ad hoc assumptions would rescue this type of mental-shift account.

Various other normalization models have been proposed to explain invariant object recognition. They cover a broad range, from applying invariant mathematical transforms (e.g., Cavanagh, 1978) to a shifter model based on the repositioning of a window of attention (Olshausen, Anderson, \& Van Essen, 1993; see also Anderson \& Van Essen, 1987). Most normalization concepts, however, evolved from psychological studies and were related to observations that RTs and/or error rates increase with the degree of transformation applied (e.g., Arnoult, 1954; Bundesen \& Larsen, 1975; Corballis, 1988; Jolicoeur, 1985; Rock, 1973; Shepard \& Metzler, 1971). Parts of these concepts are meant to explain invariance phenomena beyond visual perception (e.g., the well-known mental-rotation effect; see Shepard \& Metzler, 1971), implying that visual recognition is not yet the stage of invariance. Most of the models, however, postulate mechanisms that compensate for input translations at rather 
early processing stages, thereby allowing the patternrecognition system to detect a matching stimulus from a standard viewpoint.

Normalization of a raw image at early processing stages predicts that achieved invariance should be largely independent of the specific characteristics of a stimulus type. Difficulties for normalization hypotheses arise from the observation that increasing similarity of the patterns decreases overall performance, whereas the difference between control and displacement condition seems to be affected only marginally (Figure 7). Similarity can be detected only via the recognition process itself. Given normalization at an early processing stage, similarity should affect control and displaced conditions proportionally. This prediction, however, is not confirmed by the experimental results. Again, additional assumptions - for example, that attentional effort in the difficult task somehow slows down the shifting process-would be required to sustain normalization models. The shifter account by Olshausen et al. (1993), for example, may provide the possibility for such an adjustment by employing extensive feedback between processes that recognize the pattern and those that move a window of attention across the image. On the basis of this model, however, one would expect that manipulations facilitating or impeding the attentional shift should interfere with the displacement effect-a prediction that is not confirmed by Experiments 2-4. Facing all these problems of normalization accounts, it seems reasonable to consider alternative hypotheses for the displacement effect and its specific characteristics.

\section{Differential Effects for Same and Different Trials}

There are two ways of describing and explaining performance in same and different trials. Stated one way, performance at control locations is generally better in same than in different trials, while the opposite is often true after transfer. Even if calculation of $d^{\prime}$, the signal-detection measure of bias-free discriminability (Figure 7), rules out a superficial response bias, the information about spatial separation, though objectively irrelevant for the decision, may predispose the system to recognize "same" in control trials and "different" after translation. Biederman and Cooper (1992) offered such an explanation for similar findings in size-invariance experiments: They reported that changing the size of priming pictures has no influence on the time required for object naming. With the same set of stimuli in a same/different task, however, a systematic size dependence-specific for same

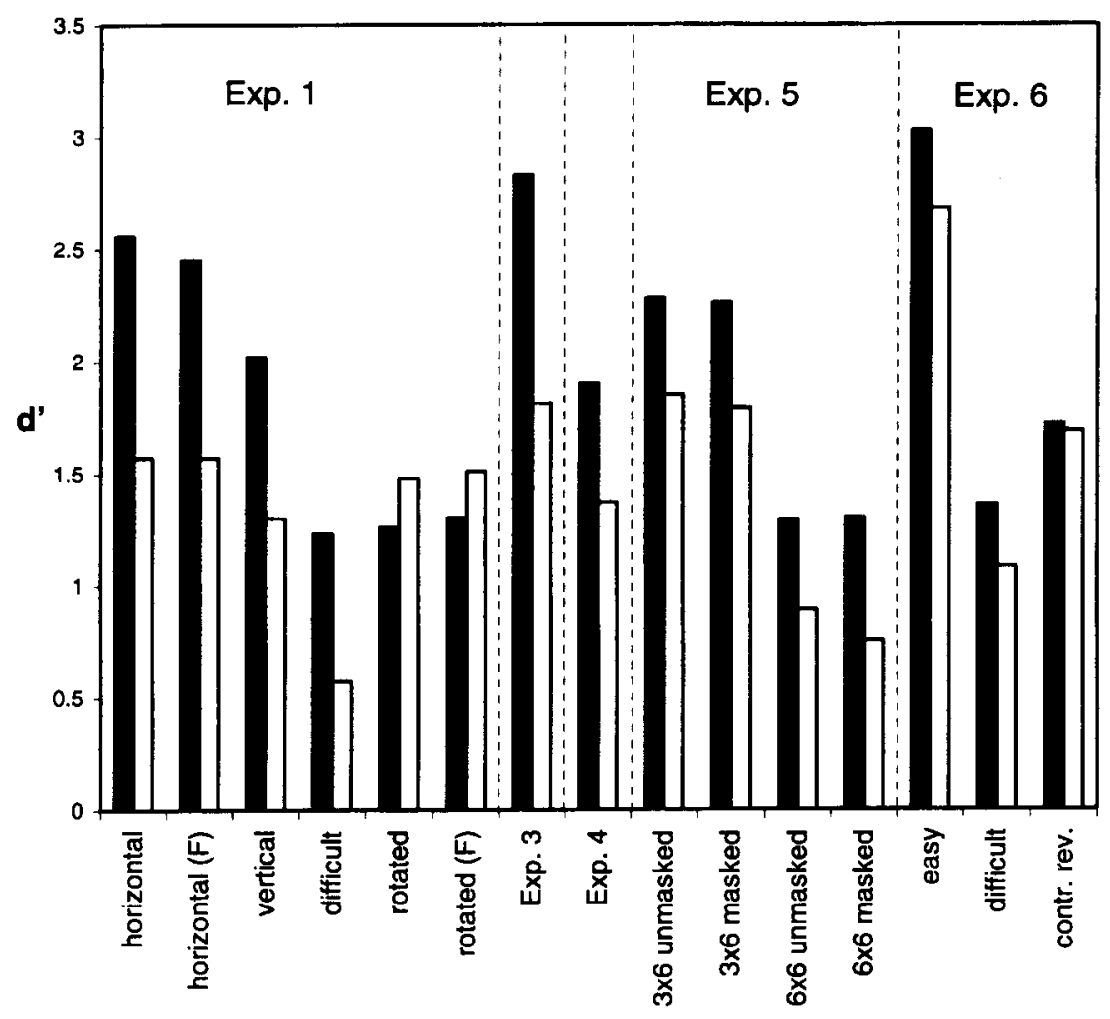

Figure 7. Discriminability $d^{\prime}$ for the most relevant data of Experiments 1 and 3-6 for control ( $0^{\circ}$ condition, black bars) and displacement trials $\left(2^{\circ}\right.$ in Experiments 1,3 , and $4 ; 4.8^{\circ}$ in Experiments 5 and 6; open bars). Horizontal, vertical, difficult, and rotated refer to Experiment 1; horizontal (F) and rotated (F) display the corresponding results from the Foster and Kahn (1985) study; $3 \times 6$ unmasked, $3 \times 6$ masked, $6 \times 6$ unmasked, and $6 \times 6$ masked are the four parts of Experiment 5; easy, difficult, and contr. rev. (contrast reversal) are the three parts of Experiment 6. 
trials-was observed for RTs and error rates. Biederman and Cooper proposed that visual shape memory itself (in the ventral processing stream) is parameter invariant, while same/different judgments are subconsciously influenced by an additional metric memory system located in the parietal cortex. According to these authors, any change in parameters such as size or position will contribute to perceptual decisions even if the object is correctly identified by an invariant shape subsystem. For this reason, they argue against same/different tasks as tools for investigating invariance of object memory.

While this two-systems hypothesis is attractive and offers some promising ways to be tested (cf. Biederman \& Cooper, 1992), one may object that the visual priming effect involves more elaborate cognitive levels beyond stages of visual recognition or memory, while perceptual matching, as in our experiments, works on levels partially specific for size, rotation, and position. That verbal or conceptual components contribute to priming is evident in that different images of the same category reduce naming latency to nearly the same level as do identical exemplars (Biederman \& Cooper, 1991, 1992; Fiser \& Biederman, 1995).

One may also ask why the brain should take the effort to separate metric and shape data if it is unable to suppress information coming from one process in decisions required from the other subsystem. Finally, the inclusion of metric position information in same/different decisions does not offer an obvious explanation for why differential same/different effects are no longer observed with reversed contrast or after stimulus rotation nor does it account for the sensitivity to translation observed in training tasks (Dill \& Fahle, 1997; Nazir \& O'Regan, 1990). We, therefore, prefer to describe and explain the differential sameldifferent effect otherwise.

\section{Limited Position Invariance in Pattern Recognition}

While same performance is strongly reduced by displacement, different trials are-with some variabilitylargely insensitive to retinal translations. This does not necessarily mean that "same" and "different" detectors rely on different perceptual mechanisms, one being position specific and the other being invariant. "Different" may, instead, simply be the default response as long as nowhere in the visual system a significant correspondence to earlier percepts is registered. The detection of "sameness" may work at a variety of processing levels employing cells with a broad range of receptive field sizes and stimulus specificities. For displaced patterns, lower levels that are more strictly tied to retinal coordinates may no longer be able to contribute to identification. This may add uncertainty to the recognition process leading to more frequent "misses" of a same pattern unless some higher level detector with a larger receptive field identifies the stimulus. Relaying information to higher levels consumes time. A decision about identity or nonidentity after translation may, therefore, be delayed, thus explaining why response latencies increase with displacement, as do error rates.
Shape perception is often hypothesized to be taking place in IT cortex. Assuming contributions to object recognition by levels lower than IT is by no means contradictory to available psychophysical and physiological data. Other visual areas with smaller average receptive field size may participate in recognition and memory. It is known, for example, that neurons in V4 are form selective (Desimone \& Schein, 1987; Kobatake \& Tanaka, 1994). A considerable amount of work on perceptual learning has revealed that even lower areas, like V1, are involved in many kinds of visual plasticity (Fahle, 1994; Fahle \& Edelman, 1993; Gilbert, 1994; Poggio, Fahle, \& Edelman, 1992; Sagi \& Tanne, 1994) and that many of these learning effects are at least partially specific to retinal location (Fahle et al., 1995; Fiorentini \& Berardi, 1981; Karni \& Sagi, 1991; Ramachandran, 1976; Shiu \& Pashler, 1992).

How would multilevel "same" detection account for the nonhomogeneous performance in different trials, which ranges from a slightly detrimental (Figure $3 b$ ) to a small incremental influence (e.g., Figure 2c) of translation? For displaced different patterns, low-level detectors should report only noise; part of the high-level detectors, however, may encounter a common feature shared by the two patterns-either accidentally or because of the similarity level set by the experimenter. For different patterns at identical or only slightly displaced locations, some low-level detectors may also report a déjà-vu. For all detector levels, the frequency of spurious recognition acts is a function of the similarity of the patterns. Whether these deceptive "microdetections" actually lead to false alarms (i.e., incorrect "same" responses when a different pattern was presented) depends mainly on the decision criterion. Determination of the latter is probably easier for the system if control and displacement trials are tested separately than if they are mixed in a session.

On the basis of the reasoning above, one might not be surprised to find variable different performance: First, false alarms should be more frequent for similar than for randomly different patterns-a rather trivial prediction that is confirmed, for example, by Experiment 6 (compare different accuracy in Figures $6 \mathrm{a}$ and $6 \mathrm{~b}$ ). Secondand less intuitive - is that one might have expected that at least for similar patterns retinal translation should reduce the incidence of spurious microdetections at lower levels. Hence, the false alarm rate should decrease (i.e., different accuracy should increase) with displacements (see Figures $2 \mathrm{c}, 4 \mathrm{~b}, 4 \mathrm{c}$, 4d, and $6 \mathrm{~b}$ for significant cases). Finally, with improved adjustment of the response criterion, the influence of spurious recognition acts should be reduced. Different performance may now even decrease with translation as is found in Experiment 3.

Translation invariance might be limited not only by small receptive fields at lower levels, but also by the required activity of many detectors with receptive fields covering overlapping, but not identical, parts of the visual field. With increasing displacements, more and more cells will lose the stimulus "out of sight," leading to increasing difficulties in distinguishing the remaining 
activity from noise. Accordingly, the error probability increases as a monotonic function of shift size. The involvement of multiple detectors also provides some understanding as to why performance with similar patterns is more sensitive to translations: In easy discrimination conditions, responses of one or a few cells may be sufficient to allow for recognition. With increasing similarity, correct "same" identification requires more detectors in order to respond adequately, leading to an additional decrease of the transfer relative to easy tasks. That some pattern types may be more sensitive to translations than others could be explained in a very similar fashion.

An abstract correspondence of stimulus design and experimental outcome corroborates our interpretation of the differential same/different effects: Rotation and contrast reversal are transformations that in our experiments affected same patterns (but not different patterns because a different pattern is different in any orientation and contrast polarity). Manipulations of similarity, on the other hand, lead to a smaller or larger difference, but do not affect the identity of a same pattern. That the displacement effect is largely specific for same trials might explain why it is affected only by the former two manipulations but not, however, by similarity variations. The influence of additional transformations can also be explained less formally: Solving the rotated and contrast reversal tasks may rely only on higher levels, where receptive fields are large, the recognized stimulus feature is relatively abstract, and invariance for other transformations is already achieved. A possible site for these abstract detectors is IT cortex, but even nonvisual cortical levels may play a role. The above reasoning - though speculative in some details-makes evident that positional specificity in same/different tasks may be explained on the basis of known characteristics of the visual pattern-recognition system. There is no need to postulate an imperfect mental-shift operation or to propose an independent, but inseparable, metric memory system.

Concerning the decision process involved, this hypothetical, post hoc model adopts a couple of properties from Krueger's (1978) noisy-operator theory (NOT). Like NOT, it relies on a single perceptual process for samel different discrimination and explains differential effects in terms of criterion adjustment relative to a continuous measure of identity. Unlike NOT, however, we suggest an identity counter that samples evidence for same rather than for different. Noise-induced spurious mismatches are replaced by a partially position-specific, multicomponent recognition system. The major advantage of this "anti-NOT" is that it offers a more concrete explanation as to how the perceptual comparison between successive images might be done and what may actually cause the uncertainty-the "noise"-in the recognition process.

\section{Distortions of the Input Image}

Finally, a potential limit for position invariance that may be less obvious and more trivial at the same time should be discussed. Retinal and cortical gradients in cell density per area of the visual field, lead to a dramatic decrease in acuity and contrast sensitivity from foveal toward peripheral vision. To counteract the reduced resolution in lateral parts of the visual field, some researchers change stimulus magnitude by a factor varying logarithmically with retinal eccentricity (cortical magnification factor; Virsu \& Rovamo, 1979). This size correction is somewhat controversial in the psychophysical literature, mostly because it is often insufficient to fully compensate for extrafoveal "shortcomings." Some authors argue that differences between peripheral and central vision are qualitative and cannot be counterbalanced by application of one single magnification factor (Jüttner \& Rentschler, 1996; Strasburger, Harvey, \& Rentschler, 1991; Strasburger, Rentschler, \& Harvey, 1994).

For our recognition tasks, we did not rescale stimuli. Plausibility considerations support our decision not to employ any correction factor. Our visual system has to cope with this problem in normal life without the external compensation of variable resolution and cortical representation. So why should compensation be employed under laboratory conditions? Besides, the main conclusions in this report would not be affected by resizing, because they are derived from experiments involving translations to isoeccentric positions, that is, to locations with the same distance from the fovea and comparable acuity. One cannot exclude, however, the possibility that gradients also influence internal distances between different pattern elements, leading to distortions varying with retinal location. The relatively small pattern size $\left(0.5^{\circ}\right.$ for dot clouds and $0.85^{\circ}$ for checkerboards) should minimize such intrastimulus distortions, but not remove them completely.

We are not aware of such object deformations, so our brains must have learned to tolerate and ignore them. But is this tolerance acquired by stimulus-specific learning (during development or by training) or by a general compensation mechanism? If position-invariant recognition of a certain pattern type is achieved by the learned mapping of local information to a collecting high-level element (e.g., Földiák, 1991), this process may produce tolerance for distortions as a side effect: The only feedback it needs is that all local detectors were stimulated by the same pattern in the outside world. Operations shifting visual input to a standard viewpoint for recognition, on the other hand, would have to compensate for deformations, too. Whether one considers distortions as a special problem of translation tolerance or not depends, therefore, very much on the model one assumes to underlie invariant object recognition.

\section{REFERENCES}

Anderson, C. H., \& VAN Essen, D. C. (1987). Shifter circuits: A computational strategy for dynamic aspects of visual processing. Proceedings of the National Academy of Sciences, 84, 6297-6301.

ArNoult, M. D. (1954). Shape discrimination as a function of the an- 
gular orientation of the stimuli. Journal of Experimental Psychology, 47, 323-328.

BACH, M. (1996) The Freiburg Visual Acuity Test-Automatic measurement of visual acuity. Optometry \& Vision Sciences, 73, 49-53.

Bagnara, S., Simion, F., \& Umiltà, C. (1984). Reference patterns and the process of normalization. Perception \& Psychophysics, 35, 186-192.

BIEDERMAN, I., \& COOPER, E. E. (1991). Evidence for complete translational and reflectional invariance in visual object priming. Perception, 20, 585-593.

Biederman, I., \& COOPER, E. E. (1992). Size invariance in visual object priming. Journal of Experimental Psychology: Human Perception \& Performance, 18, 121-133.

Bülthoff, H. H., \& Edelman, S. (1992). Psychophysical support for a two-dimensional view interpolation theory of object recognition. Proceedings of the National Academy of Sciences, 89, 60-64.

BÜLTHOFF, H. H., \& EDELMAN, S. (1993). Evaluating object recognition theories by computer graphics psychophysics. In T. A. Poggio \& D. A. Glaser (Eds.), Exploring brain functions: Models in neuroscience (pp. 139-164). New York: Wiley.

Bundesen, C., \& LARSEN, A. (1975). Visual transformation of size. Journal of Experimental Psychology: Human Perception \& Performance, 1, 214-220.

Carr, T. H., Posner, M. I., Pollatsek, A., \& Snyder, C. R. R. (1979). Orthography and familiarity effects in word processing. Journal of Experimental Psychology: General, 108, 389-414.

Cavanagh, P. (1978). Size and position invariance in the visual system. Perception, 7, 167-177.

Cerella, J. (1990). Pigeon pattern perception: Limits on perspective invariance. Perception, 19, 141-159.

Chignell, M. H., \& KRUEger, L. E. (1984). Further evidence for priming in perceptual matching: Temporal, not spatial, separation enhances the fast-same effect. Perception \& Psychophysics, 36, 257-265.

ColletT, T. S. (1992). Landmark learning and guidance in insects. Philosophical Transactions of the Royal Society of London: Series B, 337, 295-303.

Corballis, M. C. (1988). Recognition of disoriented shapes. Psychological Review, 95, 115-123.

Cronly-Dillon, J. R., Sutherland, N. S., \& Wolfe, J. (1966). Intraretinal transfer of a learned visual shape discrimination in goldfish after section and regeneration of the optic nerve brachia. Experimental Neurology, 15, 455-462.

Desimone, R., \& SCHEIN, S. J. (1987). Visual properties of neurons in area V4 of the macaque: Sensitivity to stimulus form. Journal of Neurophysiology, 57, 835-868.

DilL, M., \& FAHLE, M. (1997). The role of visual field position in patterndiscrimination learning. Proceedings of the Royal Society of London: Series $B, 264,1031-1036$.

Dill, M., \& HeISEnBerG, M. (1995). Visual pattern memory without shape recognition. Philosophical Transactions of the Royal Society of London: Series B, 349, 143-152.

Dill, M., WOLF, R., \& HeISENBERG, M. (1993). Visual pattern recognition in Drosophila involves retinotopic matching. Nature, 365, 751-753.

EDEL.MAN, S. (1995). Class similarity and viewpoint invariance in the recognition of 3D objects. Biological Cybernetics, 72, 207-220.

Edelman, S., \& BülthoFF, H. H. (1992). Orientation dependence in the recognition of familiar and novel views of three-dimensional objects. Vision Research, 32, 2385-2400.

Ellis, R., Allport, D. A., Humphreys, G. W., \& Collis, J. (1989). Varieties of object constancy. Quarterly Journal of Experimental Psychology, 41A, 775-796.

FAHLE, M. (1994). Human pattern recognition: Parallel processing and perceptual learning. Perception, 23, 411-427.

FAHLE, M., \& EDELMAN, S. (1993). Long-term learning in vernier acuity: Effects of stimulus orientation, range and of feedback. Vision Research, 33, 397-412.

Fahle, M., Edelman, S., \& Poggio, T. (1995). Fast perceptual learning in hyperacuity. Vision Research, 35, 3003-3013.

FARELL, B. (1985). "Same"-"different" judgments: A review of current controversies in perceptual comparisons. Psychological Bulletin, 98 , $419-456$
FIorentini, A., \& BeraRdi, N. (1981). Learning of grating waveform discrimination: Specificity for orientation and spatial frequency. $V i$ sion Research, 21, 1149-1158.

FISER, J., \& BIEDERMAN, I. (1995). Size invariance in visual object priming of gray-scale images. Perception, 24, 741-748.

FöLDIÁK, P. (1991). Learning invariance from transformation sequences Neural Computation, 3, 194-200.

FosTER, D. H. (1978). Visual comparison of random-dot patterns: Evidence concerning a fixed visual association between features and feature relations. Quarterly Journal of Experimental Psychology, 30, 637-654.

FosTER, D. H. (1984). Local and global computational factors in visual pattern recognition. In P. C. Dodwell \& T. Caelli (Eds.), Figural synthesis (pp. 83-115). Hillsdale, NJ: Erlbaum.

Foster, D. H. (1991). Operating on spatial relations. In R. J. Watt (Ed.), Pattern recognition by man and machine (pp. 50-68). Boca Raton, FL: CRC Press.

FOSTER, D. H., \& KAHN, J. I. (1985). Internal representations and operations in the visual comparison of transformed patterns: Effects of pattern point-inversion, positional symmetry, and separation. Biological Cybernetics, 51, 305-312.

Fujita, I., TANAKa, K., Ito, M., \& Cheng, K. (1992). Columns for visual features in monkey inferotemporal cortex. Nature, 360, 343-346.

FukUSHIMA, K. (1980). Neocognitron: A self-organizing neural network model for a mechanism of pattern recognition unaffected by shift in position. Biological Cybernetics, 36, 193-202.

GILBERT, C. D. (1994). Early perceptual learning. Proceedings of the National Academy of Sciences, 91, 1195-1197.

Heisenberg, M. (1995). Pattern recognition in insects. Current Opinion in Neurobiology, 5, 475-481.

Hubel, D. H., \& Wiesel, T. N. (1962). Receptive fields, binocular interaction and functional architecture in the cat's visual cortex. Journal of Physiology, 160, 106-154.

IRWIN, D. E. (1991). Information integration across saccadic eye movements. Cognitive Psychology, 23, 420-456.

IRWIN, J. R., \& Francis, M. A. (1995). Perception of simple and complex visual stimuli: Decision strategies and hemispheric differences in same-different judgments. Perception, 24, 787-809.

Ito, M., Tamura, H., Fujita, I., \& Tanaka, K. (1995). Size and position invariance of neuronal responses in monkey inferotemporal cortex. Journal of Neurophysiology, 73, 218-226.

JOLICOEUR, P. (1985). The time to name disoriented natural objects Memory \& Cognition, 13, 289-303.

JütT NER, M., \& RENTSCHLER, I. (1996). Reduced perceptual dimensionality in extrafoveal vision. Vision Research, 36, 1007-1022.

KAHN, J. I., \& FosTER, D. H. (1981). Visual comparison of rotated and reflected random-dot patterns as a function of their positional symmetry and separation in the field. Quarterly Journal of Experimental Psychology, 33A, 155-166.

KAHN, J. I., \& FosTER, D. H. (1986). Horizontal-vertical structure in the visual comparison of rigidly transformed patterns. Journal of Experimental Psychology: Human Perception \& Performance, 12, 422-433.

KARNI, A., \& SAGI, D. (1991). Where practice makes perfect in texture discrimination: Evidence for primary visual cortex plasticity. Proceedings of the National Academy of Sciences, 88, 4966-4970.

Kobatake, E., \& TANAKa, K. (1994). Neuronal selectivity to complex object features in the ventral visual pathway of the macaque cerebral cortex. Journal of Neurophysiology, 71, 856-867.

Kolers, P. A., Duchnicky, R. L., \& Sundstroem, G. (1985). Size in the visual processing of faces and words. Journal of Experimental Psychology: Human Perception \& Performance, 11, 726-751.

Koriat, A., Norman, J., \& Kimchi, R. (1991). Recognition of rotated letters: Extracting invariance across successive and simultaneous stimuli. Journal of Experimental Psychology: Human Perception \& Performance, 17, 444-457.

KRUEGER, L. E. (1978). A theory of perceptual matching. Psychological Review, 85, 278-304

KRUEGER, L. E. (1985). Effects of intermixed foveal and parafoveal presentation on same-different judgments: Evidence for a criterioninertia model. Perception \& Psychophysics, 37, 266-271. 
KRUEGER, L. E. (1986). Positive effect of heterogeneity of difference on the same-different disparity in letter matching. Perception \& Psychophysics, 39, 117-122.

KRUEGER, L. E., \& AlLEN, P. A. (1987). Same-different judgments of foveal and parafoveal letter pairs by older adults. Perception \& Psychophysics, 41, 329-334.

KWaK, H.-W., Dagenbach, D., \& EgeTH, H. (1991). Further evidence for a time-independent shift of the focus of attention. Perception \& Psychophysics, 49, 473-480.

Logothetis, N. K., Pauls, J., Bülthoff, H. H., \& Poggio, T. (1994). View-dependent object recognition by monkeys. Current Biology, 4, 401-414.

Logothetis, N. K., Pauls, J., \& Poggio, T. (1995). Shape representation in the inferior temporal cortex of monkeys. Current Biology, 5, $552-563$.

Macmillan, N. A., \& Creelman, C. D. (1991). Detection theory: A user's guide. Cambridge: Cambridge University Press.

MiLler, J. (1996). The sampling distribution of $d^{\prime}$. Perception \& Psychophysics, 58, 65-72.

MYERS, R. E. (1955). Interocular transfer of pattern discrimination in cats following section of crossed optic fibers. Journal of Comparative \& Physiological Psychology, 48, 470-473.

Nazir, T. A., \& O'Regan, J. K. (1990). Some results on translation invariance in the human visual system. Spatial Vision, 5, 81-100.

Olshausen, B. A., Anderson, C. H., \& Van Essen, D. C. (1993). A neurobiological model of visual attention and invariant pattern recognition based on dynamic routing of information. Journal of Neuroscience, 13, 4700-4719.

O'Regan, J. K. (1992). Solving the "real" mysteries of visual perception: The world as an outside memory. Canadian Journal of Psychology, 46, 461-488.

PHILliPs, W. A. (1974). On the distinction between sensory storage and short-term visual memory. Perception \& Psychophysics, 16, 283-290.

Poggio, T., Fahle, M., \& Edelman, S. (1992). Fast perceptual learning in visual hyperacuity. Science, 256, 1018-1021.

Proctor, R. W. (1983). A unified theory for matching-task phenomena. Psychological Review, 88, 291-326.

RaMACHANDRAN, V. S. (1976). Learning-like phenomena in stereopsis. Nature, 262, 382-384.

RentSChler, I., JÜTtNer, M., \& CAelli, T. (1994). Probabilistic analysis of human supervised learning and classification. Vision Research, 34, 669-687.

Rock, I. (1973). Orientation and form. New York: Academic Press.

RoLLs, E. T. (1992). Neurophysiological mechanisms underlying face processing within and beyond the temporal cortical visual areas. Philosophical Transactions of the Royal Society of London: Series B, 335, 11-21.
SAGI, D., \& TANNE, D. (1994). Perceptual learning: Learning to see. Current Opinion in Neurobiology, 4, 195-199.

SASLOW, M. G. (1967). Latency for saccadic eye movement. Journal of the Optical Society of America, 57, 1030-1036.

Schwartz, E. L., Desimone, R., Albright, T. D., \& Gross, C. G. (1983). Shape recognition and inferior temporal neurons. Proceedings of the National Academy of Sciences, 80, 5776-5778.

SCHWEITZER, L. R. (1991). Binary-choice decision time depends upon cerebral hemisphere and nature of task. Perceptual \& Motor Skills, 73, 147-161

ShePARD, R. N., \& METzleR, J. (1971). Mental rotation of threedimensional objects. Science, 171, 701-703

SHIU, L.-P., \& PASHLER, H. (1992). Improvement in line orientation discrimination is retinally local but dependent on cognitive set. Perception \& Psychophysics, 52, 582-588.

Strasburger, H., Harvey, L. O., JR., \& Rentschler, I. (1991). Contrast thresholds for identification of numeric characters in direct and eccentric view. Perception \& Psychophysics, 49, 495-508.

Strasburger, H., Rentschler, I., \& Harvey, L. O. (1994). Cortical magnification theory fails to predict visual recognition. European Journal of Neuroscience, 6, 1583-1588.

Tanaka, K., SaIto, H.-A., Fukada, Y., \& Moriya, M. (1991). Coding visual images of objects in the inferotemporal cortex of the macaque monkey. Journal of Neurophysiology, 66, 170-189.

ThOMPSON, P. (1980). Margaret Thatcher: A new illusion. Perception, 9, 483-484

TOVEe, M. J., Rolis, E. T., \& Azzopardi, P. (1994). Translation invariance in the responses to faces of single neurons in the temporal visual cortical areas of the alert macaque. Journal of Neurophysiology, 72, 1049-1060.

TroJe, N., \& BülthOFF, H. H. (1996). Face recognition under varying pose: The role of texture and shape. Vision Research, 36, 1761-1771.

VIRSU, V., \& Rovamo, J. (1979). Visual resolution, contrast sensitivity, and the cortical magnification factor. Experimental Brain Research, 37, 475-494.

Wachsmuth, E., Oram, M. W., \& Perrett, D. I. (1994). Recognition of objects and their component parts: Responses of single units in the temporal cortex of the macaque. Cerebral Cortex, 5, 509-522.

WEHNER, R. (1981). Spatial vision in arthropods. In H. Autrum (Ed.), Handbook of sensory physiology: Vol. 7 (Part 6, Section C, pp. 287. 616). Heidelberg: Springer-Verlag.

(Manuscript received May 16, 1996; revision accepted for publication October $28,1996$. 